\title{
Evidence of early circulation of SARS-CoV-2 in France: findings from the population-based "CONSTANCES" cohort
}

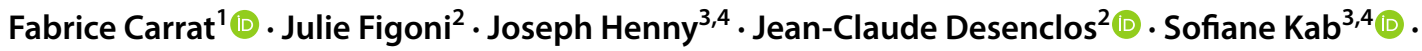 \\ Xavier de Lamballerie ${ }^{5}\left(\mathbb{C}^{-} \cdot\right.$ Marie Zins $^{3,4}$ (-)
}

Received: 4 December 2020 / Accepted: 19 December 2020 / Published online: 6 February 2021

(c) Springer Nature B.V. 2021

\begin{abstract}
Using serum samples routinely collected in 9144 adults from a French general population-based cohort, we identified 353 participants with a positive anti-SARS-CoV-2 IgG test, among whom 13 were sampled between November 2019 and January 2020 and were confirmed by neutralizing antibodies testing. Investigations in 11 of these participants revealed experience of symptoms possibly related to a SARS-CoV-2 infection or situations at risk of potential SARS-CoV-2 exposure. This suggests early circulation of SARS-CoV-2 in Europe.
\end{abstract}

Keywords SARS-CoV-2 COVID-19 · General population · Cohort

\section{Introduction}

The first identified cases of COVID-19 were detected on December 8, 2019, in Wuhan, China and the first documented case in Europe was reported retrospectively in France in one patient with a diagnosis of pneumonia and a positive SARS-CoV-2 RT-PCR result on December 27, 2019 [1]. By April 4, 2020, local community transmission was reported in all continents and over 1 million cases of COVID-19 had been confirmed worldwide. Although dedicated surveillance and contact tracing did not identify local transmission before the second half of February in Europe, there is accumulated evidence that SARS-CoV-2 circulated in early January 2020 in the East of France [2] and environmental studies suggest that the virus could have been

Fabrice Carrat

fabrice.carrat@iplesp.upmc.fr

1 APHP, Département de Santé Publique, Inserm, Institut Pierre-Louis d'Epidémiologie et de Santé Publique, Sorbonne Université, 27 rue Chaligny, 75571 Paris Cedex 12, France

2 Santé publique France, Saint-Maurice, France

3 Paris University, Paris, France

4 Inserm UMS 11, Paris Saclay University, Villejuif, France

5 Unité des Virus Emergents, Aix Marseille Univ, IRD 190, INSERM 1207, IHU Méditerranée Infection, 13005 Marseille, France present in December 2019 in Northern Italy [3]. A recent investigation of the presence of SARS-CoV-2 antibodies in 959 adults participating to a trial in Italy with blood samples collected between September 2019 to February 2020 identified $111(11.6 \%)$ samples with a positive receptorbinding protein specific enzyme-linked immunosorbent assay (ELISA), among which 4 samples collected in October, 1 in November and 1 in February were also positive in a qualitative microneutralization assay [4]. This indicates that SARS-CoV-2 could have been present in Italy since the beginning of autumn 2019. However, information on antibody responses at the early stage of the SARS-CoV-2 spread in other European countries or worldwide remains scarce.

\section{Participants and methods}

We explored the serological status for SARS-CoV-2 antibodies in participants from the "CONSTANCES" cohort. Briefly, CONSTANCES is a general population-based cohort made up of a nationwide representative sample of 215,000 adults aged 18 to 69 at inclusion. Inclusion started in 2012, and serum samples are regularly collected during the follow-up of participants for future analyses and stored in a centralized biobank. A complete description of the cohort design can be found in [5]. The cohort received ethical approval and all participants provided written consent to the cohort and additional consent to the current study. 
We selected all 9144 serum samples collected between November 4, 2019 and March 16, 2020 in participants living in the 12 mainland French regions. All samples were centralized to the virology laboratory (Unité des virus Émergents, Marseille, France) for serological analysis. The serological analysis was performed using a commercial Elisa test (Euroimmun ${ }^{\circledR}$, Lübeck, Germany) to detect anti-SARS-CoV-2 antibodies ( $\operatorname{IgG}$ ) directed against the $\mathrm{S} 1$ domain of the spike protein of the virus (ELISA-S). In accordance with the manufacturer's instructions a test was considered to be ELISA-S positive with an optical density ratio $\geq 1.1$, indeterminate between 0.8 and 1.1 , and negative, $<0.8$. All samples with an ELISA-S test $\geq 0.7$ were also tested with an in-house micro-neutralization assay to detect neutralizing anti-SARS-CoV-2 antibodies (SN), as described elsewhere [6]. A SN titer $\geq 40$ was considered to be positive. Six replicates were performed to confirm positive SN.

Participants with both ELISA-S and SN positive tests in serum sampled before February 1, 2020 were interviewed to identify potential exposure to SARS-CoV-2 infection. A trained investigator collected standardized information on clinical details (in the participant and her/his relatives), history of possible exposure (notably history of travel in Asia), and any remarkable event in close contacts (e.g. unexplained pneumonia).

\section{Results}

Participants were aged 55 (min: 19, max: 79) years at sample collection; 4623 (51\%) were female; 1503 (16\%) were living in Ile de France, 935 (10\%) in Grand-Est - these two regions being the French regions with the highest incidences of hospitalization for COVID-19 during the first semester 2020, $6706(73 \%)$ were living in the other 10 mainland regions.

Three-hundred and fifty-three $(3.9 \%)$ participants were ELISA-S positive, 138 were undetermined and 8653 were negative (undetermined and negative, 96.1\%). The proportion of ELISA-S positive increased from 1.9\% (42 of 2218) in November and 1.3\% (20 of 1534) in December to $5.0 \%$ (114 of 2268) in January, 5.2\% (114 of 2179) in February and $6.7 \%$ (63 or 945) in the first half of March $(P<0.001$, Trend test; Fig. 1).

Neutralizing antibodies were detected in $44(0.48 \%)$ participants ( 23 with a titer of 40,12 with a titer of 80,9 with a titer of 160), were undetermined in 15 participants, negative in 498 and not done in 8597 (Fig. 2). Strikingly, 13 participants with positive ELISA-S and SN tests had been sampled between November 5, 2019 and January 30, 2020. Table 1 describes the serological results in these 13 participants, among whom 11 were interviewed. Six of those interviewed did not report any symptom during the weeks preceding the sample collection. Five participants experienced signs of viral respiratory illnesses, and 8 had close contact with

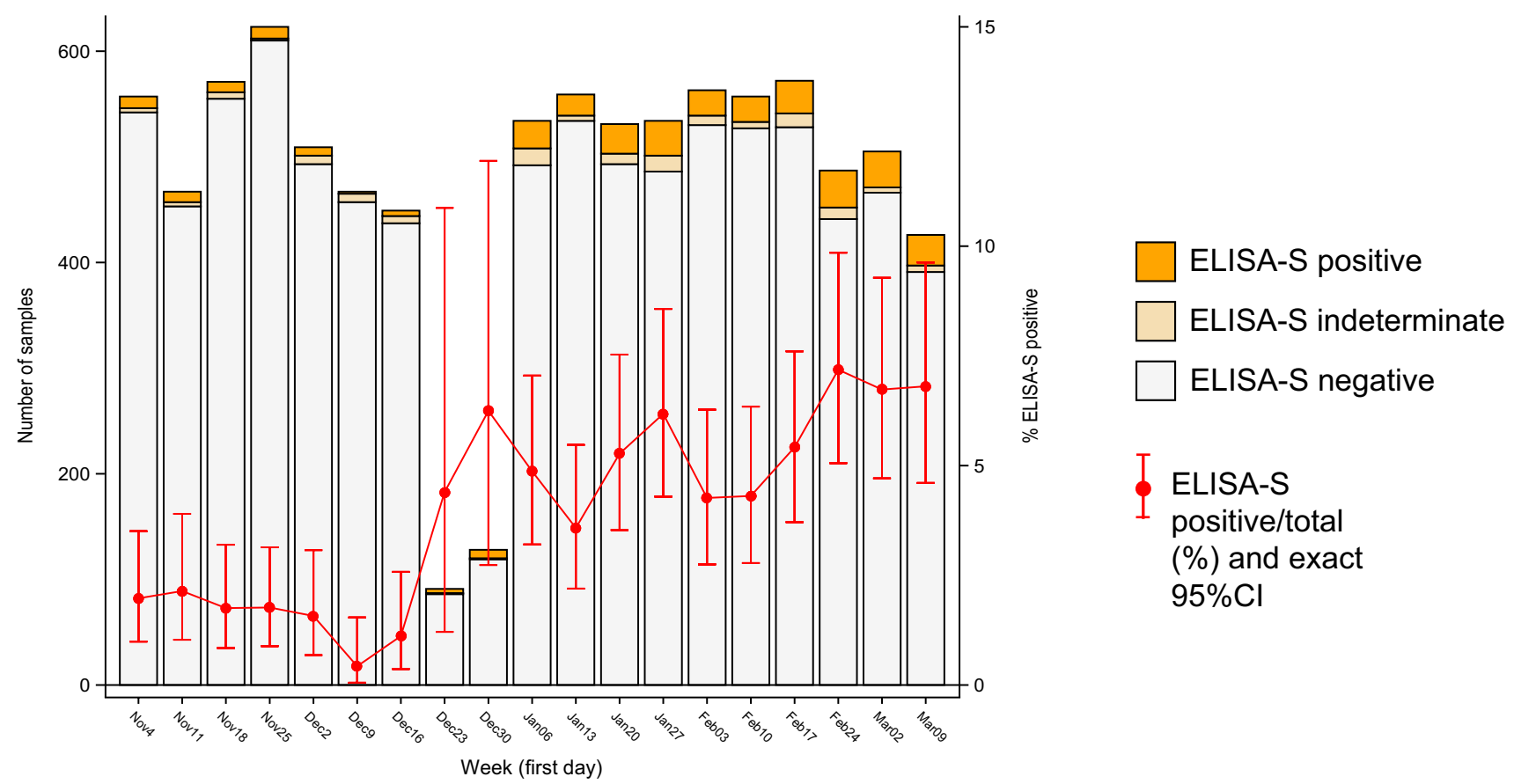

Fig. 1 Number of blood samples tested each week for anti-SARS-CoV-2 antibodies by ELISA-S IgG test (horizontal bars) and percentages of ELISA-S positive test (red dots, with exact $95 \%$ Confidence Interval) in adult participants from the CONSTANCES cohort, France 


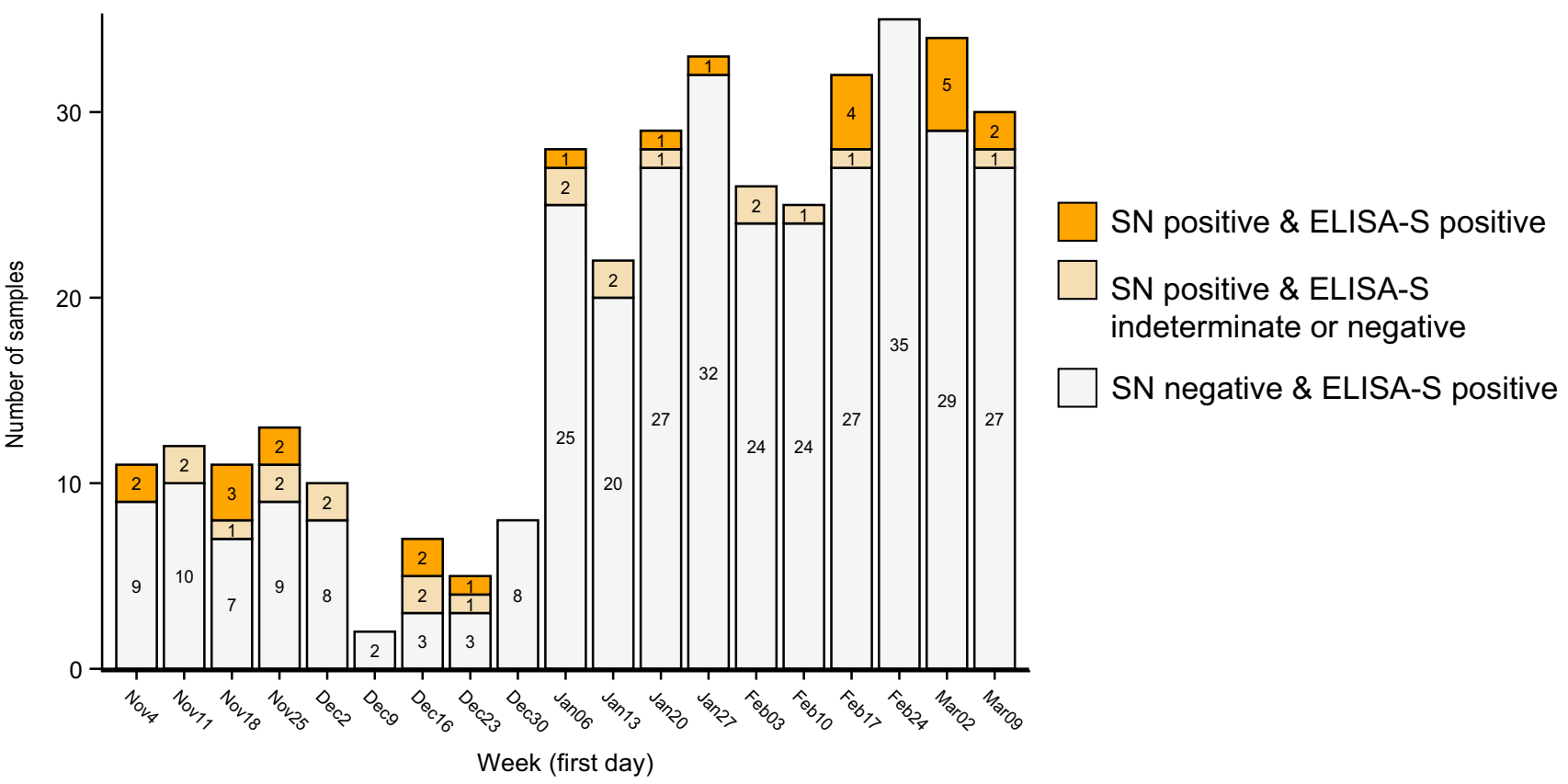

Fig. 2 Participants with a positive ELISA-S or a positive SN anti-SARS-CoV-2 test in the CONSTANCES cohort

Table 1 Participants with positive ELISA-S and positive SN on blood samples collected between November 2019 and January 2020

\begin{tabular}{|c|c|c|c|c|c|c|c|}
\hline \# & Age class (years) & Sex & $\begin{array}{l}\text { Date sample } \\
\text { (year-month) }\end{array}$ & ELISA-S & $\mathrm{SN}$ & $\begin{array}{l}\text { COVID-19 } \\
\text { symptoms }\end{array}$ & Risk factors and symptoms (travel, meetings, contact) \\
\hline 1 & $60-69$ & $\mathrm{~F}$ & 2019-11 & 1.17 & 40 & No & None \\
\hline 2 & $60-69$ & M & 2019-11 & 2.00 & 40 & No & None \\
\hline 3 & $30-39$ & $\mathrm{~F}$ & 2019-11 & 1.32 & 160 & Yes & Her partner was sick with intense cough in October 2019 \\
\hline 4 & $30-39$ & M & 2019-11 & 2.01 & 40 & NA & NA \\
\hline 5 & $40-49$ & $\mathrm{~F}$ & $2019-11$ & 1.16 & 40 & No & None \\
\hline 6 & $30-39$ & $\mathrm{~F}$ & 2019-11 & 1.75 & 80 & Yes & $\begin{array}{l}\text { Travel in Spain in early November. She had daily encounters with a } \\
\text { family member who had a respiratory illness of unknown origin } \\
\text { between October and December. She suffered from dysgeusia, } \\
\text { hyposmia, and cough before the sample was taken, but could not } \\
\text { remember the date of illness }\end{array}$ \\
\hline 7 & $30-39$ & M & 2019-11 & 2.50 & 40 & Yes & $\begin{array}{l}\text { The participant and his partner were sick with a severe cough in } \\
\text { October 2019. He had a follow-up serology at the end of July, } 2020 \text {. } \\
\text { ELISA-S }=3.82 ; \mathrm{SN}=10 \text {. The participant experienced another epi- } \\
\text { sode of cough, fever, rhinorrhea with a SARS-CoV-2 PCR positive } \\
\text { test in the second half of September } 2020\end{array}$ \\
\hline 8 & $40-49$ & M & 2019-12 & 1.53 & 160 & No & 2-month travel in Asia between October and December, 2019 \\
\hline 9 & $30-39$ & $\mathrm{~F}$ & 2019-12 & 1.88 & 40 & NA & NA \\
\hline 10 & $50-59$ & $\mathrm{~F}$ & 2019-12 & 1.83 & 80 & Yes & $\begin{array}{l}\text { Travel in Italy (Roma) end October-early November. Febrile illness at } \\
\text { the end of October } 2019\end{array}$ \\
\hline 11 & $40-49$ & $\mathrm{~F}$ & $2020-01$ & 1.71 & 40 & Yes & $\begin{array}{l}\text { Febrile illness during the third week of November } 2019 \text {. Her husband } \\
\text { and children were sick with febrile illness between November } 10 \text { and } \\
\text { November } 25,2019\end{array}$ \\
\hline 12 & $30-39$ & M & 2020-01 & 2.83 & 40 & No & Father was hospitalized for pneumonia in early December 2019 \\
\hline 13 & $40-49$ & $\mathrm{~F}$ & $2020-01$ & 1.23 & 40 & No & General Practitioner in Paris \\
\hline
\end{tabular}

NA not available 
persons who exhibited such signs or reported situations at risk of potential SARS-CoV-2 exposure. Of note, participant \#7 who was tested positive on Nov 29, 2020 had a second serological sample collected in July 2020 with a positive ELISA-S test and negative SN test-this participant also tested positive in SARS-CoV-2 RT-PCR in September 2020 for new symptoms suggesting a possible reinfection.

\section{Discussion}

This report suggests that SARS-CoV-2 infection may have occurred as early as November 2019 in France. In several participants with both ELISA-S and SN positive results, we identified symptoms, history of possible exposures, or specific events compatible with early SARS-CoV-2 infection.

The critical issue is whether these findings might result from a lack of specificity of our serological methods. The proportion of ELISA-S positive reported may look overestimated given the time period the samples were collected. We used manufacturer-defined cutoff points for ELISA-S positivity, but the test specificity and the positive predictive values can increase by using other higher cut-off values [7]. A cut-off of 2.5 was shown to be $100 \%$ specific and would have led to select only 2 positive participants out of 13 . However, all positive ELISA-S were confirmed by SN testing, and the specificity of SN was estimated at $100 \%$ over thousands of blood donors sampled in 2017-2018 when samples with a titer $\geq 40$ were considered to be positive [6]. In addition, all positive $\mathrm{SN}$ results were confirmed in multiple replicates. Thus, also we can't exclude potential misclassifications of some participants, it is unlikely that all of them were false positive results, and detailed investigation suggested compatible history of exposure in several participants. On the opposite, it should be notice that our highly specific selection of participants might lack sensitivity and have led to excluding truly infected participants or early infected participants with waning immunity. Altogether our findings are in line with the reported Italian study [4] and suggest earlier than reported SARS-CoV-2 circulation in Europe.

Acknowledgements The authors warmly thank all the volunteers of the CONSTANCES cohort. We thank Nathalie Vanhaverbeke, CEMKA, for conducting the participants interview. We thank the staff of the CONSTANCES cohort that have worked with dedication and engagement to manage the data used for this study and to ensure continuing communication with the cohort participants. In the virology department, we thank Dr. Nadège Brisbarre and the technical staff for impeccable management of samples and serological assays. In Santé publique France, we thank Harold Noel for his help building the investigation questionnaire and for his feedback on the study.

Author's contribution FC, J-CD, XdeL, MZ: study idea and design; data analysis and interpretation. JF, JH, SK, MZ: data acquisition. FC: drafting of the manuscript. All authors: critical revision of the manuscript for important intellectual content.

Funding This study The French National Research Agency [grant number ANR-10-COHO-06] Santé Publique France: N ${ }^{\circ}$ 20DMIA014-0. Cohorts funding The CONSTANCES Cohort Study is supported by the Caisse Nationale d'Assurance Maladie (CNAM), the French Ministry of Health, the Ministry of Research, the Institut national de la santé et de la recherche médicale. CONSTANCES benefits from a grant from the French National Research Agency [Grant Number ANR-11-INBS-0002] and is also partly funded by MSD, AstraZeneca, Lundbeck and L'Oreal.

Availability of data and materials In regards to data availability, data of the study are protected under the protection of health data regulation set by the French National Commission on Informatics and Liberty (Commission Nationale de l'Informatique et des Libertés, CNIL). The data can be available upon reasonable request to the corresponding author (fabrice. carrat@iplesp.upmc.fr), after a consultation with the steering committee of the CONSTANCES cohort study. The French law forbids us to provide free access to raw data; access could however be given by the steering committee after legal verification of the use of the data. Please, feel free to come back to us should you have any additional question.

\section{Compliance with ethical standards}

Conflict of interest The authors declare that there is no conflict of interests.

Ethics approval/Consent to participate The cohort received ethical approval and all participants provided written consent to the cohort and additional consent to the current study.

\section{References}

1. Deslandes A, Berti V, Tandjaoui-Lambotte Y, Alloui C, Carbonnelle $\mathrm{E}, \mathrm{Zahar} \mathrm{JR}$, et al. SARS-CoV-2 was already spreading in France in late December 2019. Int J Antimicrob Agents. 2020;55(6):106006.

2. Gerbaud L, Guiguet-Auclair C, Breysse F, Odoul J, Ouchchane L, Peterschmitt J, et al. Hospital and population-based evidence for COVID-19 early circulation in the East of France. Int J Environ Res Public Health. 2020;17(19):7175.

3. La Rosa G, Mancini P, Bonanno Ferraro G, Veneri C, Iaconelli M, Bonadonna L, et al. SARS-CoV-2 has been circulating in northern Italy since December 2019: evidence from environmental monitoring. Sci Total Environ. 2021;750:141711.

4. Apolone G, Montomoli E, Manenti A, Boeri M, Sabia F, Hyseni I, et al. Unexpected detection of SARS-CoV-2 antibodies in the prepandemic period in Italy. Tumori. 2020. https://doi. org/10.1177/0300891620974755.

5. Zins M, Goldberg M, Team C. The French CONSTANCES population-based cohort: design, inclusion and follow-up. Eur J Epidemiol. 2015;30(12):1317-28.

6. Gallian P, Pastorino B, Morel P, Chiaroni J, Ninove L, de Lamballerie X. Lower prevalence of antibodies neutralizing SARS-CoV-2 in group O French blood donors. Antiviral Res. 2020;181:104880.

7. Meyer B, Torriani G, Yerly S, Mazza L, Calame A, Arm-Vernez I, et al. Validation of a commercially available SARS-CoV-2 serological immunoassay. Clin Microbiol Infect. 2020;26(10):1386-94.

Publisher's Note Springer Nature remains neutral with regard to jurisdictional claims in published maps and institutional affiliations. 